\section{MANAGEMENT OF DEPRESSION AMONG THE ELDERLY PATIENTS IN WARD 3 GERIATRIC WARD USING THE GERIATRIC DEPRESSION SCALE (GDS)}

10.1136/bmjspcare-2011-000053.97

E Cheah, S Rajaram, H C Chua, H L Ng, H M Tim, F Fadillah, D Zheng Khoo Teck Puat Hospital, Singapore

Aim The geriatric trained nurses in Khoo Teck Puat Hospital wanted to assess the degree of depression in the elderly patients. Thus they used the geriatric Depression scale (GDS) created by Yesavage et al. to assess the patients.

Method The GDS created by Yesavage et.al (1986) has been tested and used extensively in the older population. The GDS created in 1983 had 30 questions but this was simplified in 1986 to 15 questions with scores above 5 representing depression of ascending severity. Score of $0-4$ are considered normal, complaints of 5-8 indicate mild depression, 9-11 moderate depression and 12-15 indicate severe depression. This short form was used on 12 elderly patients aged between 75-83 in Geriatric ward. The data collection was for 1 month.

Result Five of the patients had earlier referral to a psychologist. This led the psychologist to assess and find these patients moderately depressed. They received outpatient psychologist appointments. Four were found to be severely depressed and had earlier referral to psychiatrists and Medical Social workers. All 4 had to be institutionalized in the Institute of Mental Health in Singapore. All of these patients were also started on anti-depressants. 2 were found to be mildly depressed receiving a score of 5-8. Both these patients were not started on anti-depressants but were given outpatient referrals to psychologists.

Conclusion The GDS is a brief, non-somatically focused and can be either an observer or self-administered. The nurses could assess this tool easily and found it a good tool to administer. 